\title{
Optimized dispatch of wind farms with power control capability for power system restoration
}

\author{
Yunyun XIE ${ }^{1}$, Changsheng LIU ${ }^{1}$, Qiuwei WU ${ }^{2}$, Kairong LI $^{1}$, \\ Qian ZHOU $^{3}$, Minghui YIN ${ }^{1}$
}

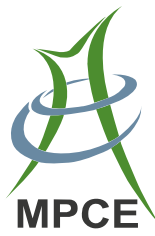

\begin{abstract}
As the power control technology of wind farms develops, the output power of wind farms can be constant, which makes it possible for wind farms to participate in power system restoration. However, due to the uncertainty of wind energy, the actual output power can't reach a constant dispatch power in all time intervals, resulting in uncertain power sags which may induce the frequency of the system being restored to go outside the security limits. Therefore, it is necessary to optimize the dispatch of wind farms participating in power system restoration. Considering that the probability distribution function (PDF) of
\end{abstract}

CrossCheck data: 15 October 2017

Received: 28 April 2017/Accepted: 15 October 2017/Published online: 17 November 2017

(C) The Author(s) 2017. This article is an open access publication

$\triangle$ Yunyun XIE

yunyun_xie@njust.edu.cn

Changsheng LIU

liucs_cq@163.com

Qiuwei WU

qw@elektro.dtu.dk

Kairong LI

likairong@njust.edu.cn

Qian ZHOU

xjtu@163.com

Minghui YIN

ymhui@vip.163.com

1 School of Automation, Nanjing University of Science and Technology, Nanjing 210094, China

2 Department of Electrical Engineering, Centre for Electric Power and Energy, Technical University of Denmark, Kgs. 2800, Lyngby, Denmark

3 Electric Power Research Institute of State Grid Jiangsu Electric Power Company, Nanjing 211103, China transient power sags is hard to obtain, a robust optimization model is proposed in this paper, which can maximize the output power of wind farms participating in power system restoration. Simulation results demonstrate that the security constraints of the restored system can be kept within security limits when wind farm dispatch is optimized by the proposed method.

Keywords Blackout, Dispatch optimization of wind farm, Power control of wind farm, System restoration,

Uncertainty of wind energy

\section{Introduction}

\subsection{Motivation and aims}

The stability of power systems is constantly increasing with the development of power system control technologies. However, the electricity market makes the operating condition of power systems closer to the operational limit. Consequently, power systems have a heightened risk of large-scale blackouts [1], which have happened several times in the past few years, such as the North American blackout in 2003 [2], the Japanese blackout in 2010 [3] and the Indian blackout in 2012 [4].

In the initial stage of power system restoration, it is necessary to restore a large number of power sources as soon as possible to improve restoration efficiency $[5,6]$. Due to the number and power limits of black-start generation units, it is difficult to accelerate system restoration by using traditional thermal units. The installed capacity of wind power has increased significantly due to environmental concerns and the need for energy supply security [7-9]. Wind farms can quickly provide power for system 
restoration after being energized, which has the potential to accelerate restoration speed of the system after a blackout. However, the volatility of wind power makes it difficult to participate in power system restoration. Accordingly, this paper proposes a strategy to handle this challenge.

\subsection{Literature overview}

In order to accelerate the speed of system restoration, some new energy sources have been employed. Battery energy storage systems (BESSs) were employed to participate in system restoration in [10]. An optimization model for power system restoration with the support of electric vehicles (EVs) is presented in [11]. However, the duration of power support from energy storage systems is limited. Additionally, very few energy storage systems have been deployed in power systems. The huge installed capacity of wind power makes it an attractive option to restore the system after a blackout. However, the output power of wind farms fluctuates when wind turbine generators (WTGs) operate in the maximum power point tracking (MPPT) mode. This fluctuating power may destabilize the fragile restored system, especially in the initial stage of system restoration. Accordingly, it is difficult for wind farms with MPPT WTGs to participate in the initial stage restoration of the power system. On the other hand, wind farms are employed to provide power in the load restoration stage $[12,13]$, because the power system by this stage is robust enough to endure the fluctuating wind power.

With the increasing wind power penetration in the power system, predictions of the output power of wind farms are collected by system operators to optimize the forward dispatch schedule for wind farms and traditional generators for maintaining system security as wind power fluctuates [14]. The overall control framework of the power system is illustrated in Fig. 1a. The wind farm control center, which receives power dispatch instructions from the system operator, further distributes power reference levels to WTG controllers, which enables the wind farm to maintain output power according to the dispatch instruction from the system operator [15-19]. Consequently, wind farms with power control capability are able to participate in the initial stage restoration of power system.

However, due to the uncertainty of wind power, when the dispatched power is greater than the minimum output power of the wind farm, the actual output power of the wind farm may not always reach the dispatched power, resulting in transient power sag [18]. An example is shown in Fig. 2. The transient power sag may result in the frequency of the restored system going outside the security limits. Therefore, it's necessary to optimize the dispatch of wind farms periodically according to the available wind power and the state of the system being restored, to ensure system security is maintained with uncertain transient power sags.

\subsection{Contribution and structure}

The main contribution of this paper is proposing a dispatch optimization model for wind farms based on robust

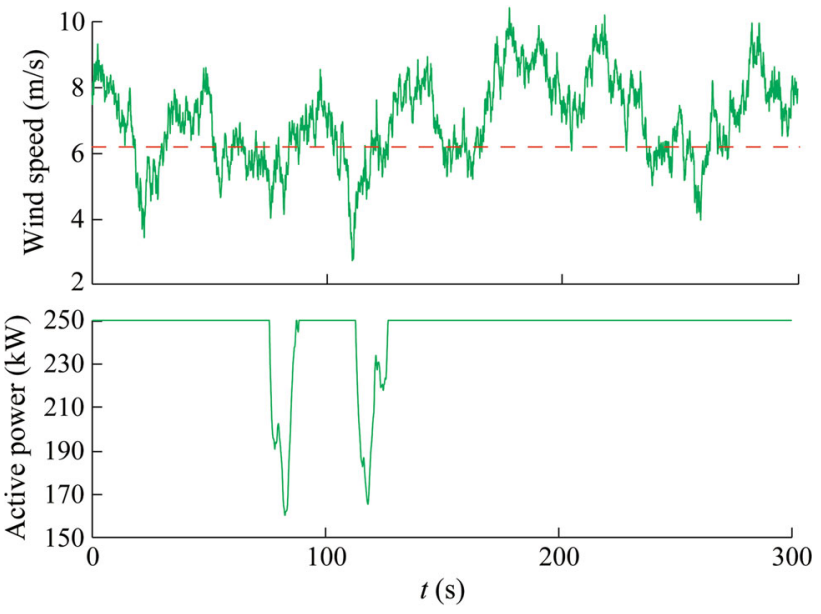

Fig. 2 Output power of wind farm with power control capability

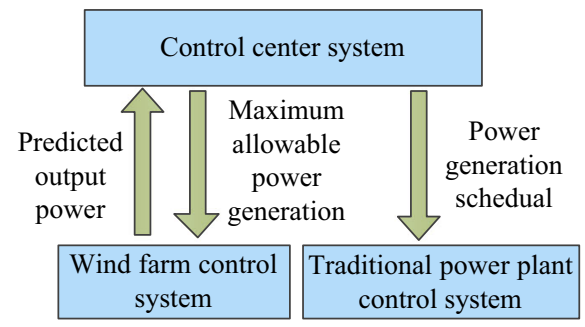

(a) Dispatch framework of power system

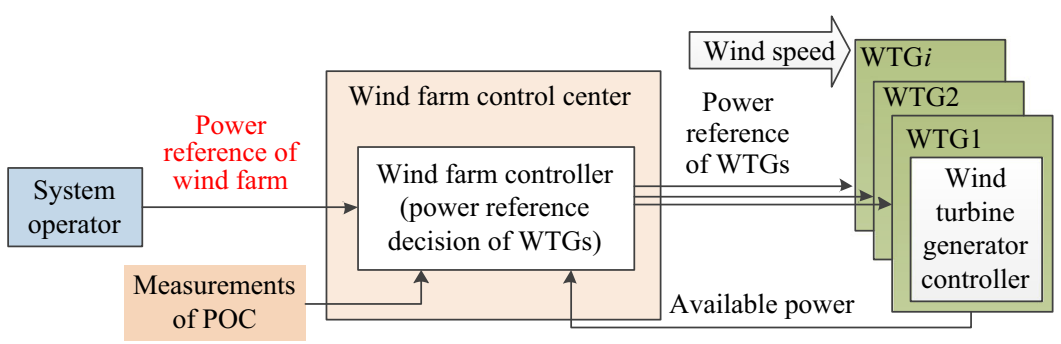

(b) Wind farm control framework for power regulation

Fig. 1 Overall control framework for power system and wind farm 
optimization to ensure system security when wind farms participate in the initial stage restoration of the power system. The advantages of the proposed method are summarized as follows.

Firstly, the wind farms with power control capability are employed to restore the power system in the initial stage, which can accelerate the speed of system restoration.

Secondly, the optimized dispatch can ensure the system security is maintained with transient power sags and power reference adjustment.

Thirdly, compared to stochastic optimization methods, the robust method employed in this paper doesn't need the probability distribution function (PDF) of transient power sags which is hard to obtain.

The paper is organized as follows: Section 2 describes the output power characteristics of wind farms participating in power system restoration. Section 3 presents the robust optimization model for determining the forward dispatch schedule of wind farms. Case studies are presented and discussed in Section 4, followed by conclusions.

\section{Output power characteristics of wind farms with power control capability}

Because the available output power of wind farms is determined by wind speed, the dispatched power of wind farms for system restoration must be less than the maximum available output power.

According to the wind speed, the operating regime of WTGs can be divided into four parts: the starting area, MPPT area, constant speed area and constant power area. The maximum available output power of WTGs at different wind speeds is:

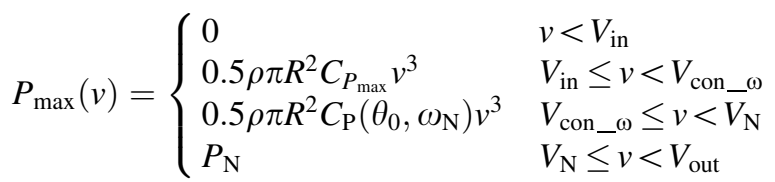

where $v$ is the wind speed; $V_{\text {in }}$ is the cut-in speed; $V_{\text {con_ } \omega}$ is the upper limit of wind speed in the MPPT area; $V_{\mathrm{N}}$ is the upper limit of wind speed in the constant speed area; $V_{\text {out }}$ is the cut-out speed for WTG operation; $\rho$ is the air density; $R$ is the radius of the wine turbine rotor; $C_{\mathrm{pmax}}$ is the maximum utilization coefficient of wind power; $\theta_{0}$ is the initial pitch angle; $P_{\mathrm{N}}$ is the maximum output power of the wind generator.

When the predicted wind speed is $v_{\text {pre }}$, the dispatched power of the wind farm is limited by the maximum available output power:
$0 \leq P_{\text {ref }} \leq n P_{\max }\left(v_{\text {pre }}\right)$

where $n$ is the number of WTGs in the wind farm, and $P_{\text {ref }}$ is the power reference of the wind farm dispatched by system operator.

Due to the uncertainty and intermittency of wind power, when the dispatched power is less than the actual minimum output power, the output power of wind farm will be constant; when the predicted average output power is greater than the actual average output power, there will be a transient power sag; when the predicted average output power is between the actual average output power and the actual minimum output power, there may be a power sag which is related to the wind conditions. During power system restoration, a transient power sag represents uncertainty: it is difficult to precisely predict the start time and the duration of transient power sags. Therefore, it's necessary to optimize the dispatched power to ensure the security of the restored system.

\section{Robust optimization model of dispatched power}

In this section, a robust optimization model [20, 21] is proposed to optimize the dispatched power of wind farms, in order to ensure the security of the restored system.

\subsection{Formulating a robust optimization model for power dispatch optimization}

The control center periodically collects the predicted output power of wind farms to optimize the power generation schedule which will be sent back to them. Because the PDF of transient power sags of wind power is difficult to obtain, it is difficult to employ common methods to deal with the uncertainty of transient power sags such as the stochastic methods or fuzzy methods [22, 23]. Robust optimization, which does not need the PDF or membership function of uncertain variables, has been widely applied to the optimization of power dispatch [14], emission dispatch [20], and other tasks.

Therefore, a robust optimization model is employed for optimizing the power dispatched from wind farms in this paper. It can be formulated as follows:

$$
\left\{\begin{array}{c}
\max f(\boldsymbol{y} *) \\
\text { st. } H(\boldsymbol{y}) \leq \boldsymbol{C} \\
\boldsymbol{y} \in[\underline{y}, \boldsymbol{y} *]
\end{array}\right.
$$

where $\boldsymbol{y}^{*}$ denotes the dispatched power of wind farms; $\boldsymbol{y}$ is the minimum output power; $\boldsymbol{y}$ denotes the actual output power fluctuating in the range between the minimum output power and the dispatched power; $H(\boldsymbol{y}) \leq \boldsymbol{C}$ denotes constraints of wind farms participating in system 
restoration, such as power flow constraints and the transient frequency constraint.

\subsection{Detailed robust power dispatch optimization model}

\subsubsection{Objective function}

For initial stage restoration of the power system, it is necessary for wind farms to provide as much power as possible to accelerate system restoration. Because the output power of wind farms will be equal to the dispatched power most of the time, the objective function of the robust power reference optimization can be expressed as:

$f=\max \left\{\min \sum_{i=1}^{m} P_{\text {ref_}} i\right\}$

where $f$ is the total output power of wind farms; $m$ is the number of wind farms; and $P_{\text {ref }_{-} i}$ is the dispatched power of wind farm $i$.

\subsubsection{Constraints}

For robust optimization, the feasible solution under the worst-case scenario can guarantee the security of other scenarios. Therefore, some of the following constraints represent worst-case scenarios that should be satisfied to ensure the system security.

1) Maximum available output power constraint. According to the output power model of wind farms in Subsection 2.1, the dispatched power cannot exceed the maximum available output power:

$0 \leq P_{\text {ref } \_i} \leq n_{i} P_{\text {max } \_i}\left(v_{\text {pre } \_i}\right)$

where $n_{i}$ is the number of WTGs in the wind farm $i$; $v_{\text {pre } \_i}$ is the predicted wind speed in the wind farm $i$; and $P_{\max _{-} i}$ is the maximum output power model of wind farm $i$.

2) The constraint on transient frequency deviation by transient power sags. A transient power sag will result in a transient frequency deviation which should be kept in the safe range. Suppose that the wind farms are in the same area and the effects of wind speed on each wind farm are identical, then the transient power sag of each wind farm can be added up for the worst-case scenario. The resulting transient frequency constraint is:

$\sum_{j=1}^{m}\left(P_{\mathrm{ref} \_j}-P_{\min \_j}\right) / \sum_{i=1}^{n G} \frac{P_{\mathrm{G} i}}{d f_{i}} \leq \Delta f_{\max }$ where $n_{\mathrm{G}}$ is the number of conventional generation units; $P_{\mathrm{G} i}$ is the active output power of unit $i$ in the restored system; $P_{\min _{\_} j}$ is the minimum output power of wind farm $j ; \triangle f_{\max }$ is the maximum variation allowed which is $0.5 \mathrm{~Hz}$ in this paper; and $d f_{i}$ is the transient frequency response coefficient of unit $i$ [21]. The minimum output power of wind farm $j$ can be formulated as:

$P_{\text {min } \_j}=(1-\alpha) P_{\text {ave } \_j}$

where $P_{\text {ave } j}$ is the predicted average output power of wind farm, and $\alpha$ is the fluctuation range of output power.

3) Power flow constraint. The power flow needs to be balanced:

$$
\left\{\begin{array}{l}
P_{i}+P_{\mathrm{ref} \_i}=P_{L i}+V_{i} \sum_{j=1}^{N} V_{j}\left(G_{i j} \cos \delta_{i j}+B_{i j} \sin \delta_{i j}\right) \\
Q_{i}=Q_{L i}+V_{i} \sum_{j=1}^{N} V_{j}\left(G_{i j} \sin \delta_{i j}-B_{i j} \cos \delta_{i j}\right)
\end{array}\right.
$$

where $P_{i}$ and $Q_{i}$ are the active and reactive power injection at node $i ; P_{L i}$ and $Q_{L i}$ are the active and reactive load at node $i ; V_{i}$ is the voltage of node $i ; G_{i j}$ and $B_{i j}$ are the conductance and susceptance between nodes $i$ and $j ; \delta_{i j}$ is the phase-angle difference between $V_{i}$ and $V_{j}$; and $N$ is the number of nodes.

The output power of conventional units and the voltage of every node need to be within the permissible range:

$$
\left\{\begin{array}{l}
P_{\mathrm{Gimin}} \leq P_{\mathrm{G} i} \leq P_{\mathrm{Gimax}} \\
Q_{\mathrm{Gimin}} \leq Q_{\mathrm{G} i} \leq Q_{\mathrm{G} i \max } \\
V_{j \min } \leq V_{j} \leq V_{j \max }
\end{array}\right.
$$

where $P_{\mathrm{G} i}$ and $\mathrm{Q}_{\mathrm{G} i}$ are the active and reactive output power of conventional unit $i$, respectively; $P_{\text {Gimax }}$ and $P_{\mathrm{G} i m i n}$ are the maximum and minimum active power; $Q_{\text {Gimax }}$ and $Q_{\text {Gimin }}$ are the maximum and minimum reactive power; $V_{j}$ is the voltage of node $j$; and $V_{j \max }$ and $V_{j \min }$ are the maximum and minimum voltage of node $j$.

4) The constraint on transient frequency deviation by dispatched power adjustment. Besides the transient power sags of the output power, the dispatched powers of wind farms are adjusted periodically according to the available wind power and the status of the system being restored. Periodic power adjustment by the dispatched power variation also has a great impact on the frequency of the restored system which may 
exceed limits. The constraint on transient frequency deviation caused by the periodic power adjustment $\sum \triangle P_{\text {ref } \_j}$ is:

$\sum_{j=1}^{n} \Delta P_{\text {ref } \_j} / \sum_{i=1}^{n_{G}} \frac{P_{G i}}{d f_{i}} \leq \Delta f_{\max }$

The solutions of the proposed model are the maximum dispatched powers of wind farms which can ensure system security even with transient power sags.

\subsection{Solution of proposed model}

The robust optimization models developed above are nonlinear and hard to solve. Intelligent algorithms are commonly employed to solve them. In this paper, the artificial bee colony algorithm is employed to solve the optimization model due to its good performance on searching efficiency [24].

\section{Case study}

\subsection{Test system}

The IEEE 39-bus system is used to verify the efficiency of the proposed model. The population size, maximum cycle number and predetermined number of cycles in the artificial bee colony algorithm are set as 20, 100 and 5, respectively.

The topology of the IEEE 39 bus system is shown in Fig. 3. As indicated, it is assumed that the power plants located at node 3, 4, 16, 23, 26, 27, 29 are wind farms, and that thermal units are located at nodes 30-39. The unit located at node 30 is the black-start unit, and others are non-black-start units. The restoration path of all units during system restoration is given in Table 1 , as well as the capacity of generators and loads at each node.

\subsection{Optimal result of proposed model}

The system after node 33 is restored is employed to verify the proposed robust method, and this is marked with bold-face font in Table 1 and blue lines in Fig. 3. A dispatch decision needs to be made at the moment when the output power of units at node 37 is $51.2 \mathrm{MW}$, and units at node 33, 38 and 39 have been restarted but not been connected to the power grid. The capacities of restored loads at node 25, 26, 29, 39, 27 and 16 are $60 \mathrm{MW}, 50 \mathrm{MW}$, $30 \mathrm{MW}, 106 \mathrm{MW}, 96 \mathrm{MW}$ and $136 \mathrm{MW}$, respectively. The predicted average power of each wind farm and the dispatched power at the current moment are listed in Table 2.

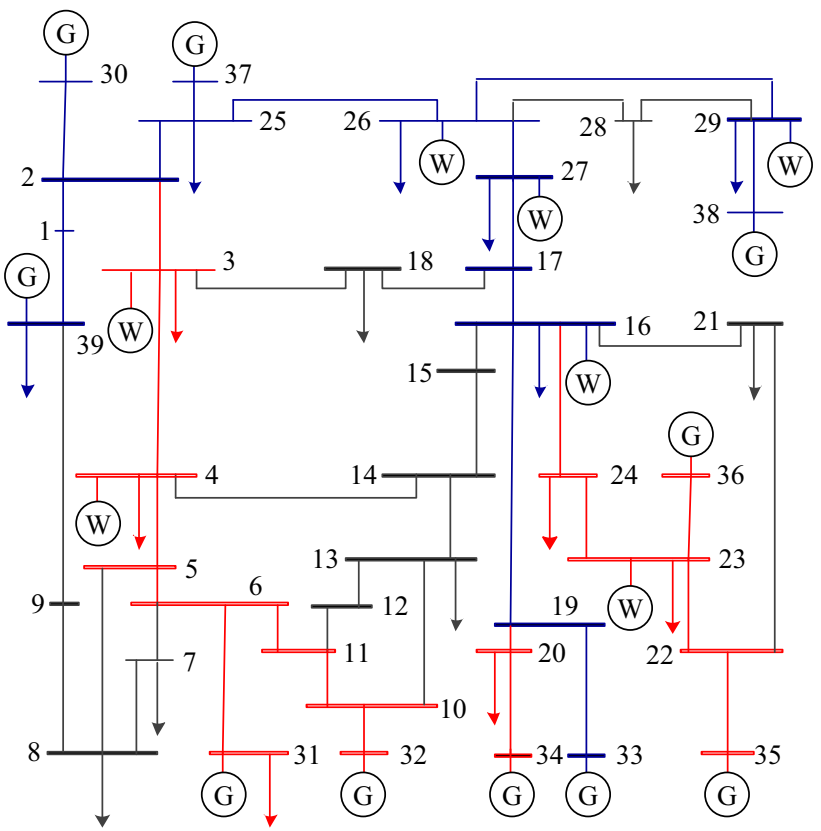

Fig. 3 IEEE 39 bus system under restoration

When transient power sags are not considered, the expected optimal dispatched powers for the wind farms are 76.5 MW, 88.5 MW, 90 MW, and 120 MW, respectively, and the total power output of wind farms is $375 \mathrm{MW}$. Considering transient power sags, the robust optimization model is solved for different fluctuation ranges $\alpha$, which result in different dispatched powers listed in Table 3.

The relationship between the fluctuation range and optimal values of dispatched power are obtained and shown in Fig. 4. It can be observed that there are negative correlations between $\alpha$ and the total dispatched power. When the wind power fluctuation range is less than 0.2 , the actual fluctuating output power is still within security limits and the optimal result of the robust optimization model is similar to the result of the deterministic model. As $\alpha$ increases, the likelihood of transient power sags will induce the restored system to go over security limits and the dispatched powers of wind farms need to decrease to reduce the range of transient power sags.

\subsection{Comparison between different methods}

The dispatched powers determined by the deterministic model (DM), the fuzzy chance constrained model (FCCM) [23] and the robust optimization model (ROM) are compared in this section. In the DM, transient power sags are ignored. The confidence level and risk coefficient of the model are set as 0.6 and 0.4 , respectively. Because a fuzzy model of transient power sags is hard to obtain, 5 experimental groups of parameters listed in Table 4 are chosen to select the best 
Table 1 Restoration path of all units during system restoration

\begin{tabular}{|c|c|c|c|}
\hline No. & Node id & Capacity of unit (MW) & Capacity of load (MW) \\
\hline 1 & 30 & 350 & 0 \\
\hline 2 & 2 & 0 & 0 \\
\hline 3 & 25 & 0 & 224 \\
\hline 4 & 37 & 640 & 0 \\
\hline 5 & 26 & 240 & 139 \\
\hline 6 & 29 & 200 & 283.5 \\
\hline 7 & 38 & 930 & 0 \\
\hline 8 & 1 & 0 & 0 \\
\hline 9 & 39 & 1100 & 1104 \\
\hline 10 & 27 & 200 & 843 \\
\hline 11 & 17 & 0 & 0 \\
\hline 12 & 16 & 160 & 329 \\
\hline 13 & 19 & 0 & 0 \\
\hline 14 & 33 & 750 & 0 \\
\hline 15 & 20 & 0 & 680 \\
\hline 16 & 34 & 660 & 0 \\
\hline 17 & 3 & 150 & 322 \\
\hline 18 & 4 & 180 & 1000 \\
\hline 19 & 5 & 0 & 0 \\
\hline 20 & 6 & 0 & 0 \\
\hline 21 & 31 & 1145 & 9.2 \\
\hline 22 & 24 & 0 & 462.9 \\
\hline 23 & 23 & 150 & 495 \\
\hline 24 & 36 & 660 & 0 \\
\hline 25 & 11 & 0 & 0 \\
\hline 26 & 10 & 240 & 0 \\
\hline 32 & 32 & 750 & 0 \\
\hline 28 & 22 & 0 & 0 \\
\hline 29 & 35 & 750 & 0 \\
\hline
\end{tabular}

Table 2 Dispatched and predicted output power of wind farms

\begin{tabular}{lrrrr}
\hline No. of wind farm & 16 & 26 & 27 & 29 \\
\hline Current dispatched power & 75 & 70 & 100 & 90 \\
Predicted average output power & 100 & 80 & 120 & 100
\end{tabular}

fuzzy parameter. The fuzzy parameters of group 5 are the most conservative, having the lowest total dispatched wind power, so these are selected for simulation.

In this simulation, the fluctuation range of wind power is $30 \%$, and the total output power of wind farms is fluctuating randomly in the range of $280 \mathrm{MW}$ to $520 \mathrm{MW}$. The dispatched power values solved by the three methods are shown in Table 5. The actual wind power fluctuation is randomly generated 20 times to verify the effectiveness of the three methods. Simulation results are shown in Fig. 5, where the brown line shows the results of the DM, the blue
Table 3 Optimal results for different fluctuation range of wind power

\begin{tabular}{lllrll}
\hline$\alpha$ & \multicolumn{3}{l}{ Output power } & \multicolumn{2}{c}{ Total output power (MW) } \\
\cline { 2 - 4 } & 16 & \multicolumn{1}{c}{26} & \multicolumn{1}{c}{27} & \multicolumn{1}{c}{29} & \\
\hline 0 & 76.5 & 88.5 & \multicolumn{1}{c}{90} & \multicolumn{1}{c}{120} & 375.0 \\
0.1 & 97.0 & 74.6 & 92.6 & 110.5 & 374.7 \\
0.2 & 94.4 & 71.9 & 119.9 & 88.4 & 374.6 \\
0.3 & 89.1 & 63.2 & 87.7 & 106.3 & 346.3 \\
0.4 & 79.1 & 59.3 & 74.8 & 93.2 & 306.3 \\
0.5 & 59.2 & 58.0 & 66.3 & 82.9 & 266.4 \\
0.6 & 50.4 & 41.1 & 83.3 & 51.4 & 226.2 \\
0.7 & 50.0 & 38.2 & 47.3 & 50.9 & 186.3 \\
0.8 & 31.4 & 32.9 & 52.3 & 29.9 & 146.5 \\
0.9 & 25.8 & 20.5 & 33.7 & 26.4 & 106.4 \\
\hline
\end{tabular}

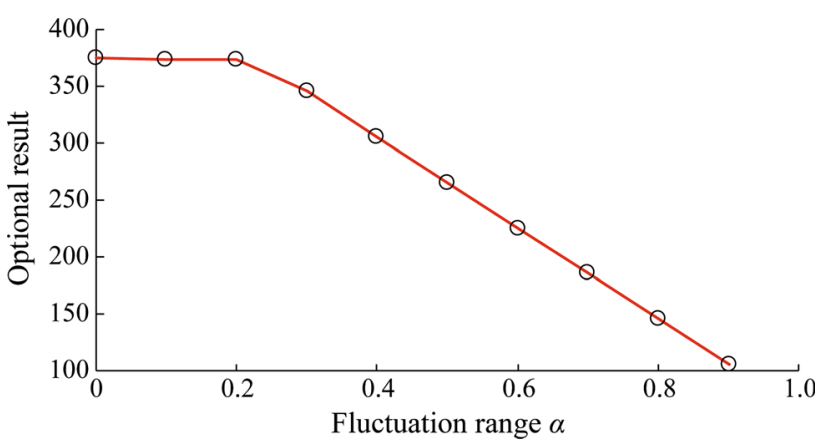

Fig. 4 Relationship between $\alpha$ and optimal result

Table 4 Optimal results for different fuzzy parameters

\begin{tabular}{|c|c|c|c|c|c|}
\hline Group no. & 1 & 2 & 3 & 4 & 5 \\
\hline \multicolumn{6}{|l|}{ Fuzzy parameter } \\
\hline$\beta$ & 0.6 & 0.6 & 0.6 & 0.6 & 0.6 \\
\hline$\lambda$ & 0.4 & 0.4 & 0.4 & 0.4 & 0.4 \\
\hline$\mu_{1}$ & 0.5 & 0.6 & 0.7 & 0.5 & 0.2 \\
\hline$\mu_{2}$ & 0.6 & 0.9 & 0.8 & 0.6 & 0.4 \\
\hline$\mu_{3}$ & 1 & 1.2 & 0.9 & 0.7 & 0.6 \\
\hline$\mu_{4}$ & 1.1 & 1.3 & 1 & 0.8 & 0.8 \\
\hline \multicolumn{6}{|l|}{ Power reference } \\
\hline 16 & 81 & 97.5 & 99 & 102 & 102 \\
\hline 26 & 90 & 121.5 & 91.5 & 96 & 88.5 \\
\hline 29 & 121.5 & 100.5 & 117 & 94.5 & 105 \\
\hline 27 & 82.5 & 84 & 64.5 & 73.5 & 69 \\
\hline Total output power & 375 & 403.5 & 372 & 366 & 364.5 \\
\hline
\end{tabular}

line shows the results of the FCCM and the green line shows the results of the ROM. For the DM and the FCCM, the total output power in some cases is 0 , indicating that restored system can't satisfy the security limits. There are 5 
Table 5 The dispatched power values solved by three methods

\begin{tabular}{llllll}
\hline $\begin{array}{l}\text { No. of wind } \\
\text { farms }\end{array}$ & $\begin{array}{l}16 \\
(\mathrm{MW})\end{array}$ & $\begin{array}{l}26 \\
(\mathrm{MW})\end{array}$ & $\begin{array}{l}27 \\
(\mathrm{MW})\end{array}$ & $\begin{array}{l}29 \\
(\mathrm{MW})\end{array}$ & $\begin{array}{l}\text { Total } \\
\text { dispatch } \\
\text { power }\end{array}$ \\
\hline $\begin{array}{l}\text { Current power } \\
\text { reference }\end{array}$ & 75 & 70 & 100 & 90 & 335 \\
$\begin{array}{l}\text { Dispatch power } \\
\text { DM }\end{array}$ & 76.5 & 88.5 & 90 & 120 & 375 \\
FCCM & 102.0 & 69.0 & 105.0 & 88.5 & 364.5 \\
ROM & 89.0 & 63.2 & 87.7 & 106.3 & 346.3 \\
$\begin{array}{l}\text { Dispatch power adjustment } \\
\text { DM }\end{array}$ & +1.5 & +18.5 & -10 & +30 & +40.0 \\
FCCM & +27.0 & -1.0 & +5.0 & -1.5 & +29.5 \\
ROM & +14 & -6.8 & -2.3 & +16.3 & +11.3 \\
\hline
\end{tabular}

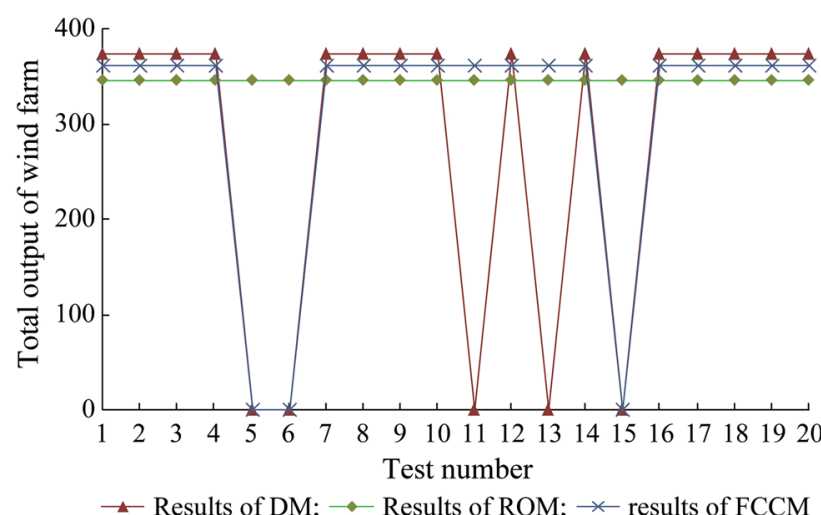

Fig. 5 Total output power of wind farms solved by three models

Table 6 Three cases of deterministic model violating frequency limit

\begin{tabular}{lrrr}
\hline Case no. & \multicolumn{1}{r}{2} & \multicolumn{1}{c}{3} \\
\hline$\alpha$ & -0.23 & -0.25 & -0.29 \\
Minimum total output power (MW) & 308 & 300 & 288 \\
If there is security problem & & & \\
DM & $\sqrt{ }$ & $\sqrt{ }$ & $\sqrt{ }$ \\
FCCM & $\times$ & $\times$ & $\sqrt{ }$
\end{tabular}

RO

cases exceeding security limits for the DM and 2 cases for the FCCM. For the robust model, the security of the restored system is always maintained.

In Table 6, three cases are selected to illustrate the problem of the DM and the FCCM. The maximum frequency deviation is set as $0.5 \mathrm{~Hz}$ during system restoration [21] and the maximum allowable power variation is 66.34 MW for the restored system. From Tables 5 and 6, we can infer that the dispatched power adjustments for each wind farm are all less than the maximum allowable power variation. However, the transient power sags for the DM are 67.0 MW, 75.0 MW, and 87.0 MW respectively in the 3 selected cases. They all exceed the maximum allowable power variation. The transient power sags for the FCCM are 56.5 MW, 64.5 MW, and 76.5 MW respectively. The transient power sag for the $3^{\text {rd }}$ case exceeds the maximum allowable power variation. The transient power sags for the ROM are 38.3 MW, 46.3 MW, and 58.3 MW, which are all within the safe range. From these results, we can conclude that although some potential wind power output is not used for the ROM, the restored system is maintained within security limits through transient power sags of wind power.

\subsection{Effect of wind power participating in system restoration}

This section focuses on the effect of wind farms used for system restoration. The whole restoration path is listed in Table 1, as well as the capacity of units and loads at each node. The thermal units, which require time to heat boilers, can't immediately provide power for the restored system when it is energized, while the wind farms with power control capability can promptly supply power for the restored system after being energized. The critical minimum and maximum intervals of thermal unit restoration are not considered in this example, however, the restoration time of each transmission line is $4 \mathrm{~min}$, which means the total restoration time of all units is $112 \mathrm{~min}$ due to this constraint alone. In the restoration process, the dispatched power levels of wind farms are optimized by the robust model every $5 \mathrm{~min}$ according to the states of the system being restored and the predicted wind speed. The total dispatched power of wind farms is illustrated in Fig. 6.

From Table 1, it can be seen that the wind farm at node 26 is energized first at $20 \mathrm{~min}$, and its capacity is $240 \mathrm{MW}$. The dispatched power of the wind farm is constrained by the ability of the restored system to withstand power fluctuations. As the output power of the wind farms increases, the likelihood of transient power sags becomes the main constraint for the optimization of the dispatched power. Taking the output power at $40 \mathrm{~min}$ as an example, although the capacity of energized wind farms is $640 \mathrm{MW}$, the output power of wind farms is only $220 \mathrm{MW}$ to mitigate the impact of transient power sags. The ability of the restored system to withstand power fluctuation is gradually improved due to energized thermal units connecting to the restoring system, for example, the total output power of wind farms is improved at 32 min because of the thermal units in node 37 connected to the restored system.

The effect of wind farms participating in system restoration is shown in Fig. 7. Compared with the system 


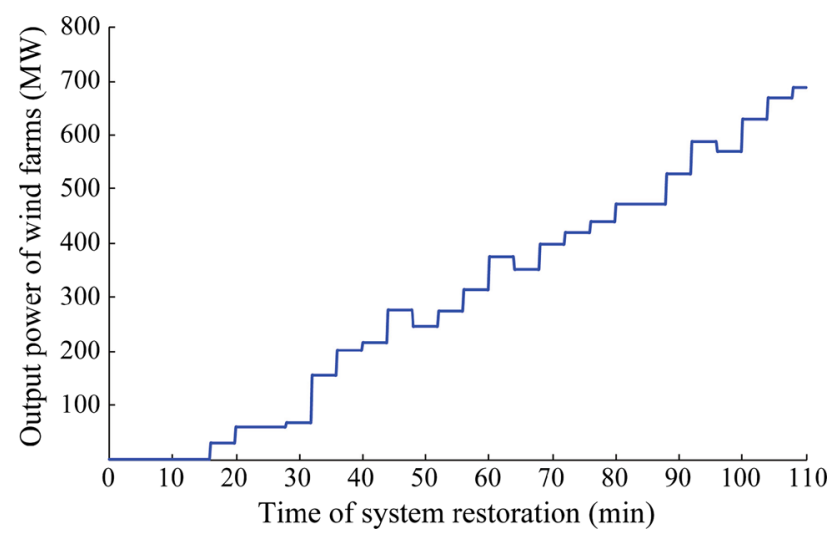

Fig. 6 Total output power of wind farms in the process of system restoration

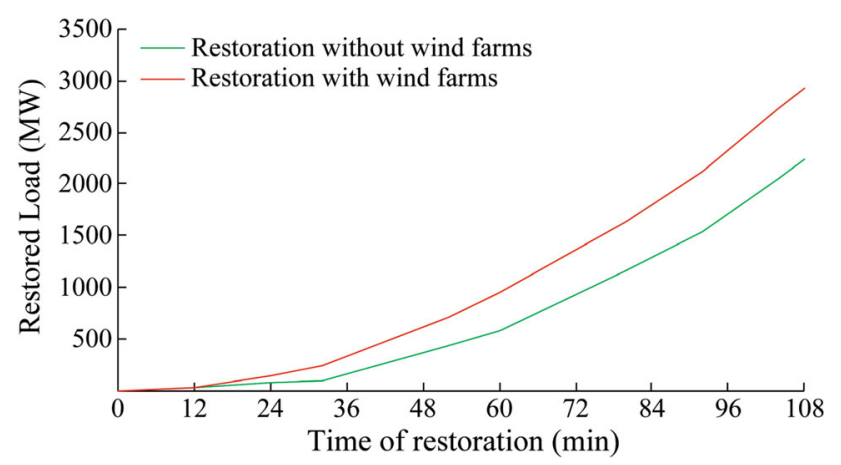

Fig. 7 Comparison of different system restoration strategies

restoration without wind farms, the system restoration with wind farms picks up more loads, especially in the initial stage of system restoration. Half of the restored power is supplied by wind farms at $40 \mathrm{~min}$, and $30 \%$ of the energy is supplied by wind farms in the process of restoration. Because the losses of critical loads become more severe as the duration of the interruption increases, the extra power supplied by wind farms is crucial for critical loads in the initial stage of system restoration.

\section{Conclusion}

Setting proper dispatched power levels for wind farms is important and challenging for wind farms participating in system restoration. Due to the uncertainty of wind power, the dispatched power of wind farms may not always be followed, which results in transient power sags. In addition, any adjustment of dispatched power will also cause power variation. These two kinds of power fluctuations cause transient frequency deviations in the restored system which may exceed security limits. The probability density function of transient power sags is hard to obtain, so it is not a trivial task to set the dispatched power of wind farms during system restoration.

This paper proposes a robust optimization model to determine optimal dispatched power levels for wind farms and solves it using the artificial bee colony algorithm. Simulated case study results based on the IEEE 39 bus system illustrate that system restoration with the dispatched powers for wind farms determined by the proposed method can satisfy the security constraints in the presence of output power fluctuations. Compared with the deterministic model, although part of the available wind power is curtailed, the proposed model can ensure the security of the restored system. Wind power makes a significant contribution, accelerating the restoration speed, and suppling $30 \%$ of the total energy during system restoration.

Acknowledgement This work was supported by the National Natural Science Foundation of China (No. 51507080), the Science and Technology Project of State Grid Corporation of China (5228001600DT).

Open access This article is distributed under the terms of the Creative Commons Attribution 4.0 International License (http:// creativecommons.org/licenses/by/4.0/), which permits unrestricted use, distribution, and reproduction in any medium, provided you give appropriate credit to the original author(s) and the source, provide a link to the Creative Commons license, and indicate if changes were made.

\section{References}

[1] Liu YT, Fan R, Terzija V (2016) Power system restoration: a literature review from 2006 to 2016. J Mod Power Syst Clean Energy 4(3):332-341. https://doi.org/10.1007/s40565-016-02192

[2] Wu FF, Moslehi K, Bose A (2005) Power system control centers: past, present, and future. Proc IEEE 93(11):1890-1908

[3] Zhang Q, Ishihara KN, Mclellan BC et al (2012) Scenario analysis on future electricity supply and demand in Japan. Energy 38(1):376-385

[4] Xue Y, Xiao S (2013) Generalized congestion of power systems: insights from the massive blackouts in India. J Mod Power Syst Clean Energy 1(2):91-100. https://doi.org/10.1007/s40565-0130014-2

[5] Hou Y, Liu CC, Sun K et al (2011) Computation of milestones for decision support during system restoration. IEEE Trans Power Syst 26(3):1399-1409

[6] Sun W, Liu CC, Zhang L (2011) Optimal generator start-up strategy for bulk power system restoration. IEEE Trans Power Syst 26(3):1357-1366

[7] Tan Z, Ngan HW, Wu Y et al (2013) Potential and policy issues for sustainable development of wind power in China. J Mod Power Syst Clean Energy 1(3):204-215. https://doi.org/10.1007/ s40565-013-0037-8

[8] Daraeepour A, Kazempour SJ, Patiño-Echeverri D et al (2016) Strategic demand-side response to wind power integration. IEEE Trans Power Syst 31(5):3495-3505 
[9] Simão HP, Powell WB, Archer CL et al (2017) The challenge of integrating offshore wind power in the US electric grid. Part II: simulation of electricity market operations. Renew Energy 103:418-431

[10] Liu W, Sun L, Lin Z et al (2016) Multi-objective restoration optimisation of power systems with battery energy storage systems. IET Gener Transm Distrib 10(7):1749-1757

[11] Sun L, Wang X, Liu W et al (2016) Optimisation model for power system restoration with support from electric vehicles employing battery swapping. IET Gener Transm Distrib 10(3):771-779

[12] El-Zonkoly AM (2014) Renewable energy sources for complete optimal power system black-start restoration. IET Gener Transm Distrib 9(6):531-539

[13] Sun L, Wang X, Liu W et al (2016) Optimisation model for power system restoration with support from electric vehicles employing battery swapping. IET Gener Transm Distrib 10(3):771-779

[14] Wu W, Chen J, Zhang B et al (2014) A robust wind power optimization method for look-ahead power dispatch[J]. IEEE Trans Sustain Energy 5(2):507-515

[15] Hansen AD, Sørensen P, Iov F et al (2006) Centralised power control of wind farm with doubly fed induction generators. Renew Energy 31(7):935-951

[16] Zhao H, Wu Q, Guo Q et al (2015) Distributed model predictive control of a wind farm for optimal active power controlpart II: implementation with clustering-based piece-wise affine wind turbine model. IEEE Trans Sustain Energy 6(3):840-849

[17] Rabelo BC, Hofmann W, da Silva JL et al (2009) Reactive power control design in doubly fed induction generators for wind turbines. IEEE Trans Ind Electron 56(10):4154-4162

[18] Zhao H, Wu Q, Wang J et al (2017) Combined active and reactive power control of wind farms based on model predictive control. IEEE Trans Energy Convers 32(3):1177-1187

[19] Yin M, Xu Y, Shen C et al (2016) Turbine stability-constrained available wind power of variable speed wind turbines for active power control. IEEE Trans Power Syst 32(3):2487-2488

[20] Liu X, Xu W (2010) Minimum emission dispatch constrained by stochastic wind power availability and cost. IEEE Trans Power Syst 25(3): 1705-1713

[21] Adibi MM, Borkoski JN, Kafka RJ et al (1999) Frequency response of prime movers during restoration. IEEE Trans Power Syst 14(2):751-756

[22] Constantinescu EM, Zavala VM, Rocklin M et al (2011) A computational framework for uncertainty quantification and stochastic optimization in unit commitment with wind power generation. IEEE Trans Power Syst 26(1):431-441

[23] Miranda V, Hang PS (2005) Economic dispatch model with fuzzy wind constraints and attitudes of dispatchers. IEEE Trans Power Syst 20(4):2143-2145
[24] Karaboga D, Akay B (2009) A comparative study of artificial bee colony algorithm. Appl Math Comput 214(1):108-132

Yunyun XIE received his Ph.D. degree in control theory and engineering from Nanjing University of Science and Technology, Nanjing, China, in 2013. He is currently an Assistant Professor with the Department of Smart Grid Information Engineering, Nanjing University of Science and Technology. His major research interests include power system restoration and power system transient stability.

Changsheng LIU received his M.E. degree from Chongqing University, Chongqing, China, in 2001. Presently he is pursuing Ph.D. degree in Nanjing University of Science and Technology, Nanjing, China. His research interests include power system restoration and optimization.

Qiuwei WU received his Ph.D. degree in power system engineering from Nanyang Technological University, Singapore, in 2009. He has been an Associate Professor since September 2013 with the Centre for Electric Technology, Department of Electrical Engineering, Technical University of Denmark, Kgs. Lyngby, Denmark. His research interests include smart grid operation and optimization.

Kairong LI received his B.E. degree from Xuzhou University of Technology, XuZhou, China, in 2014. Presently he is pursuing M.E. degree in Nanjing University of Science and Technology, Nanjing, China. His research interests include power system restoration and optimization.

Qian ZHOU received his M.E. degree from Xi'an Jiaotong University, Nanjing, China, in 2005. He is currently working in Jiangsu Electric Power Research Institute. His major research interests include power system dispatch and power system transient stability.

Minghui YIN received his Ph.D. degree in control science and engineering from the Nanjing University of Science and Technology, Nanjing, China, in 2009. He is currently an Associate Professor with the School of Automation, Nanjing University of Science and Technology. His major research interests include wind power conversion system and power system transient stability. 